\title{
MEMS Electrostatic Micro-Power Generator for Low Frequency Operation
}

\author{
P. D. Mitcheson * P. Miao B. H. Stark E. M. Yeatman A. S. Holmes T. C. Green \\ Department of Electrical and Electronic Engineering, Imperial College London, U.K.
}

\begin{abstract}
This paper describes the analysis, simulation and testing of a microengineered motion-driven power generator, suitable for application in sensors within or worn on the human body. Micro-generators capable of powering sensors have previously been reported, but these have required high frequency mechanical vibrations to excite a resonant structure. However, bodydriven movements are slow and irregular, with large displacements, and hence do not effectively couple energy into such generators. The device presented here uses an alternative, non-resonant operating mode. Analysis of this generator shows its potential for the application considered, and shows the possibility to optimise the design for particular conditions. An experimental prototype based on a variable parallel-plate capacitor operating in constant charge mode is described which confirms the analysis and simulation models. This prototype, when precharged to $30 \mathrm{~V}$, develops an output voltage of $250 \mathrm{~V}$, corresponding to $0.3 \mu \mathrm{J}$ per cycle. The experimental test procedure and the instrumentation are also described.
\end{abstract}

Key words: Micro-generator, self-powered sensors, motion-to-electric energy conversion

\section{Introduction}

Several examples of motion-driven micro-generators capable of powering microelectronic circuits have been reported $(1 ; 2 ; 3 ; 4 ; 5 ; 6 ; 7)$. Some potential applications for micro-generators are wearable, medical electronics and remote sensing. These generators can break the finite energy restriction of battery powered devices by harvesting energy, in the form of motion, from their environment. The reported examples use a mass-spring system which resonates when the frame of the device is vibrated. The motion of the mass relative to the frame is damped by one of several

\footnotetext{
* Corresponding author

Email address: pmitchesoneiee.org (P. D. Mitcheson).
}

energy conversion mechanisms, namely electromagnetic force $(1 ; 2 ; 5)$, electrostatic force $(3 ; 6 ; 7)$, or piezoelectric force (4). The different force-movement characteristics of these conversion mechanisms give different operating characteristics for the generators (8). Thus there are velocity-damped resonant generators (VDRG) based on electromagnetic damping, and coulomb-damped resonant generators (CDRG) based on electrostatic damping. Each form of generator suits a particular type of operation and each can be optimised to some extent to match given operating conditions. The resonant nature of these devices gives them some common properties. The generators are most effective when a steady single frequency excitation is available that matches closely the resonant frequency. The desired physical size of micro-generators restricts 
the resonant frequency to above the order of $100 \mathrm{~Hz}$. A vibration source such as engine vibration would fall into this range and is thus a suitable source for such a resonant generator. Further, higher frequency vibrations are small in amplitude, and the resonant action amplifies this so that larger displacements can be used in the damping mechanism.

Whilst there are clear advantages in eliminating battery storage or wired power connections from wearable and implantable electronics, these applications present a particular challenge for energy harvesting. The motion of limbs or the cardio-vascular system are at low, non-constant frequency, and it is not feasible to construct a highly miniaturised mechanically resonant structure for operation at circa $1 \mathrm{~Hz}$ as required. Further, the amplitude of body-driven motion of the frame is likely to be larger than the displacement limit of the mass within the frame (given a MEMS implementation), so resonant amplification is not possible. Thus a non-resonant motion of the mass is required, and this should ideally be obtained without a sprung suspension, as any spring force may easily overwhelm inertial forces. Figure 1 shows a generic model for the generators, with $x(t)$ and $y(t)$ giving the absolute motion of the proof mass and the frame respectively, $z(t)$ the internal (relative) motion, and $Z_{l}$ the maximum internal displacement. The mass, spring and energy extracting damping force are represented by $m, k$ and $f$ respectively. In (8), detailed analyses and comparisons are presented of the VDRG and CDRG, and of a third generator type, the non-resonant Coulomb Force Parametric Generator (CFPG). Using this analysis, figure 2 has been plotted to show which of three competing architectures yields the greatest power for a range of frame-motion amplitudes $Y_{0}$, for an example of a $0.5 \mathrm{~g}$ mass with a maximum internal displacement $Z_{l}$ of $250 \mu \mathrm{m}$. The two resonant alternatives provide the highest energy for low source amplitudes, the VDRG being best above resonant frequency and the CDRG best just below resonant frequency. Significantly below resonant frequency, or when the framemotion amplitude exceeds the maximum travel of the mass by one or more orders of magnitude, the CFPG yields the highest energy.

This paper reports on the further development of this non-resonant form of generator. Analysis is developed to enable an electrostatic non-resonant generator to be designed and optimised. Fabrication of a pro-

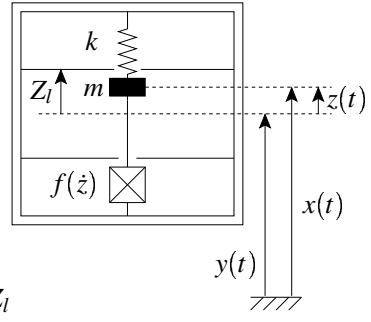

Fig. 1. Generic model of motion-driven electrical generator.

totype generator is described, and verification of the analysis against experimental testing of the prototype is provided.

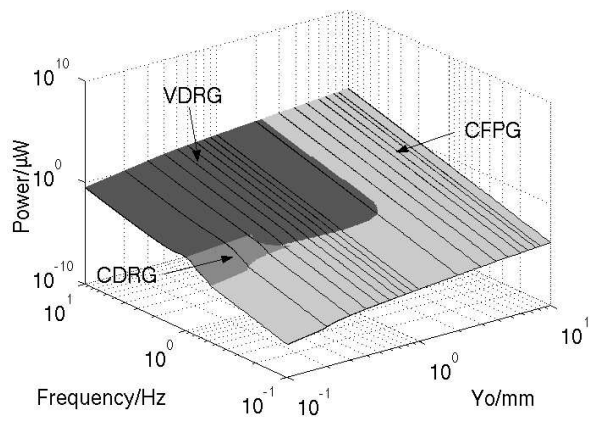

Fig. 2. Theoretically achievable output power vs. frequency and amplitude of source motion, for the generator architecture yielding the highest power at each operating point (as indicated), with $Z_{l}=250 \mu \mathrm{m}, \mathrm{m}=0.5 \mathrm{~g}$ and a resonant frequency of $1 \mathrm{~Hz}$.

\section{Coulomb-force parametric generator}

In all three generator architectures, acceleration of the frame by the external system creates a force $f$ on the mass. The product of this force and the relative displacement, $f d z$, represents work done on the damper and suspension, and the integral of $f d z$ over a complete cycle represents the converted energy per cycle. In the resonant cases, the work can be absorbed by the damping mechanism or stored in the spring, from where it can be released to the damper at a different part of the motion cycle. In a non-resonant device, however, work is only done on the damper, so to maximise the output power this damper force should be maximised through the whole of the relative motion. In addition, the damping force must be less than $m \ddot{y}$ in order for the mass to break away from the frame. There- 
fore the maximum energy can be obtained if the mass moves during the peak of acceleration of the source motion. If this is done, the optimal damping force will be approximately constant at just below $m \ddot{y}_{\text {max }}$.

The CFPG is a non-resonant generator and a specific instance of figure 1 , where the spring constant $k$ is zero and the damping force $f$ has a constant value, and always opposes the relative motion between the mass and frame. Such a damping force is known as a Coulomb force (friction between moving surfaces is an example of a Coulomb force). This constant force can be implemented electrically as the attractive force between parallel capacitor plates moving normally to each other with a constant charge, or by parallel sliding plates (or comb-drive electrodes) operated in constant voltage. The CFPG is not tuned to a resonant frequency; instead energy conversion only starts when the acceleration of the frame is greater than the damping (or holding) force per unit of proof-mass, thus causing the mass to move relative to the frame. The motion is one in which the mass tends to move from one end of the frame to the other at the peak of the acceleration of the frame. If the external motion is harmonic, then $\ddot{y}_{\text {max }}=\omega^{2} Y_{0}$. As shown in (8), the power generated by an ideal CFPG is the force-distance product for the damper, multiplied by twice the frequency of operation, assuming generation in both directions per cycle, which will now be:

$P=2 \beta\left(\frac{Z_{0}}{Y_{0}}\right) \frac{Y_{0}^{2} \omega^{3} m}{\pi}$

where $\beta$, the breakaway factor, is the fraction of the maximum acceleration at which the mass is able to move relative to the frame, and $Z_{0}$ is the relative distance moved.

An optimal value of $\beta$, corresponding to a maximum force-distance product for the damper, should be chosen. As $\beta$ increases, the maximum distance that the mass moves relative to the frame will decrease. A possible analytic procedure for calculating the optimal value of $\beta$ is to write the equation of motion of the mass relative to the frame, and by differentiation find the time at which the distance is a maximum because, assuming the damper is realised by a capacitor, this is the point at which the energy stored in it is a maximum and should be extracted. The value could then be substituted into the equation of motion and an optimal force-distance product found. Unfortunately, the derivative with respect to $t$ of the equation of motion has no closed-form solution, and consequently no analytic solution for an optimal $\beta$ is available. However, some deductions about optimal $\beta$ can be made by writing the equation of uniformly accelerated motion. In particular, it can be shown (8) that the optimal $\beta$ value is defined solely in terms of the ratio $\frac{Z_{l}}{Y_{0}}$ for sinusoidal input motion.

Time-domain simulations were run for the CFPG, varying $\beta$. These show that for small relative displacement limits, the optimal value of $\beta$ for maximum power generation is that which allows the mass to just move the full distance, $2 Z_{l}$. For values of $\frac{Z_{l}}{Y_{0}}$ above $0.566, \beta$ must be lowered more quickly than what is optimal for maximum power generation per stroke if power is extracted in both directions, because of the need to change direction within half a cycle. For $\frac{Z_{l}}{Y_{0}}$ above 1.335 , this causes the energy per stroke to be less than half that for single-sided operation (power extracted in one direction only), and so single-sided operation gives greater total power. In the single-sided case, although the power stroke can last longer than half a cycle, the mass must return to its starting point within a full cycle for periodic operation, and this requirement limits the $\beta$ value to 0.34 for $\frac{Z_{l}}{Y_{0}}>1.16$.

A plot of optimal $\beta$ values is shown in figure 3 . Figure 3(a) shows optimal values for single-sided and double-sided operation, whilst 3(b) shows the optimal value and operating mode to use, assuming that the device can change between operating in double-sided or single-sided mode.

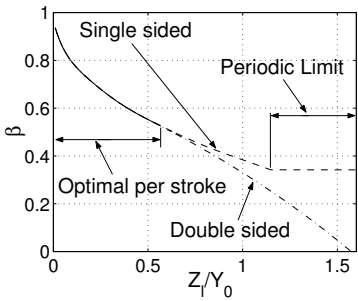

(a) Optimal $\beta$ for single and double sided motion.

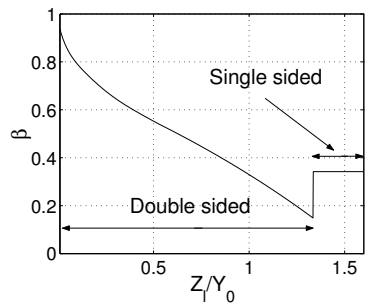

(b) $\beta$ for optimal energy generation.
Fig. 3. Optimal $\beta$ values.

For comparison, the maximum power for a VDRG, 
in the case where the maximum internal displacement constrains the available power, is given in (8) as:

$P_{\text {max }} c Z=Y_{0}^{2} \omega^{3} m \frac{1}{2 \omega_{c}^{2}}\left(\frac{Z_{l}}{Y_{0}}\right)^{2} \sqrt{\omega_{c}^{4}\left(\frac{Y_{0}}{Z_{l}}\right)^{2}-\left(1-\omega_{c}^{2}\right)^{2}}$

where $\omega_{c}$ is the ratio of operating to resonant frequency. It can be seen that the power enhancement at resonance $\omega_{c}=1$ is diminished if an amplitude magnification cannot be achieved. For small $\frac{Z_{l}}{Y_{0}}$ values, (2) reduces to:

$P_{\text {res }}=\frac{1}{2} Y_{0}^{2} \omega^{3} m \frac{Z_{l}}{Y_{0}}$,

i.e., approximately the on-resonance value. (For very small $\omega_{c}$ values, the motion is not displacement constrained so (2) does not apply, but an equivalent result can be obtained). Taking the ratio of (1) and (3), we obtain

$P_{C F P G} / P_{V D R G}=4 \beta / \pi$

This indicates that the CFPG can generate more power than resonant generators where the optimal $\beta$ value is above $\pi / 4$; this corresponds to a displacement limit $\frac{Z_{l}}{Y_{0}}$ of 0.107 . The power output for an ideal CFPG is shown in figure 4 , normalised to $m \omega^{3} Y_{0}{ }^{2}$.

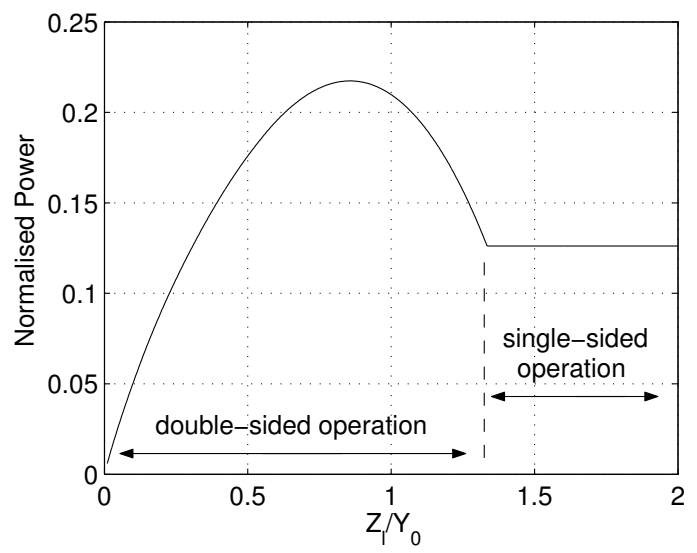

Fig. 4. Ideal performace of CFPG.

\subsection{Practical constraints}

Constant charge and constant voltage mode electrostatic generators have been compared in (3), where the latter is shown to provide significantly higher output. However, this is based on the assumption that the initial and final capacitances $C_{1}$ and $C_{2}$, and the maximum voltage in the system $V_{m}$, are the limiting factors. In such a case, and assuming $C_{1} \gg C_{2}$, a constant voltage device generates $\frac{1}{2} C_{1} V_{m}{ }^{2}$ per stroke, while a constant charge device produces only $\frac{1}{2} C_{2} V_{m}{ }^{2}$. However, the ultimate limit is achieved by setting the holding force just below $m \ddot{y}_{\text {max }}$ as described above, so an optimal device would have a higher starting capacitance in the constant charge case than in the constant voltage case. Furthermore, a higher initial capacitance is easier to achieve for parallel plates pulled apart (which gives constant force in the constant charge case) than for plates sliding over each other, because in the former case a small gap can be set by physical stops, while in the latter case it must be maintained over the whole range of travel by the suspension. High capacitance is also difficult to achieve for comb drives.

With these considerations in mind, a constant charge mode design using parallel plates pulled apart was chosen, with the initial and final separations set by mechanical stops. For such a device, the main constraint on optimal device operation is likely to be the voltage capability of the output side power-processing circuitry. However, it is beneficial to maximise the starting capacitance in order to reduce the voltage at which the correct holding force is obtained, and thus reduce the precharge voltage.

\section{Electro-mechanical simulations}

Due to the small quantities of charge involved for power generation in the CFPG, it is important to accurately simulate the electronic components. Parasitic capacitances in the order of $10 \mathrm{pF}$ in the input stage of the control electronics connected to the moving electrode would absorb the charge and cancel out the generating effect of the moving electrode. Similarly, leakage currents would drain the generated energy. Consequently, an integrated electro-mechanical system simulation must be capable of incorporating precise models of specifically designed semiconductor devices, in order to accurately specify the power extraction circuitry. SPICE is well established for this kind of task, and in addition it is capable of modelling a system 
written as a set of differential equations, and this is the approach that has been taken to produce an integrated electro-mechanical simulation.

One key component required in the simulation of the CFPG is a variable capacitor, which, although not a native SPICE component, can be successfully modelled as a fixed capacitor which models the stored charge and has a separation dependant voltage source. Results of an integrated electromechanical simulation, figure 5 , illustrate the operation of the CFPG in single-sided mode, i.e., the only electrostatic force present in the system acts to pull the mass towards the bottom of the frame. Because the simulated device operates at constant charge, the capacitor plate voltage is proportional to the relative distance between the moving capacitor plates. In the upper plot the dashed lines show the motion of the upper and lower stops of the generator frame, whilst the solid line shows the absolute motion of the moving plate. The lower plot shows the voltage on the moving plate. The features of precharge, breakaway and discharge can clearly be seen. Note that for this optimal trajectory, the relative velocity drops to zero as the top contact is approached.
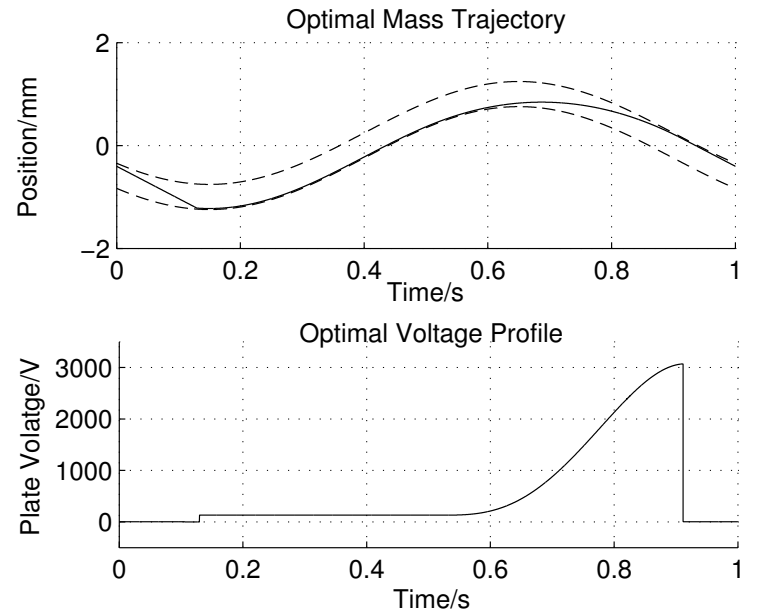

Fig. 5. Optimal performance of CFPG.

\section{Test Setup and Instrumentation}

Figure 6 shows the experimental setup used for evaluating the prototype generator. The generator and associated electronics were mounted on a shaker table, and the position of this was continually monitored with a linear variable displacement transducer for low frequency motion and an accelerometer for high frequency motion. The shaker was orientated with its axis horizontal to minimise the effects of gravity on the proof mass. Charge was applied to the capacitor plates by means of a variable DC voltage source connected between the grounded bottom electrode and the precharging contact. The moving plate was connected permanently to a voltage probe circuit, and at the position of minimum capacitance to a discharge circuit.

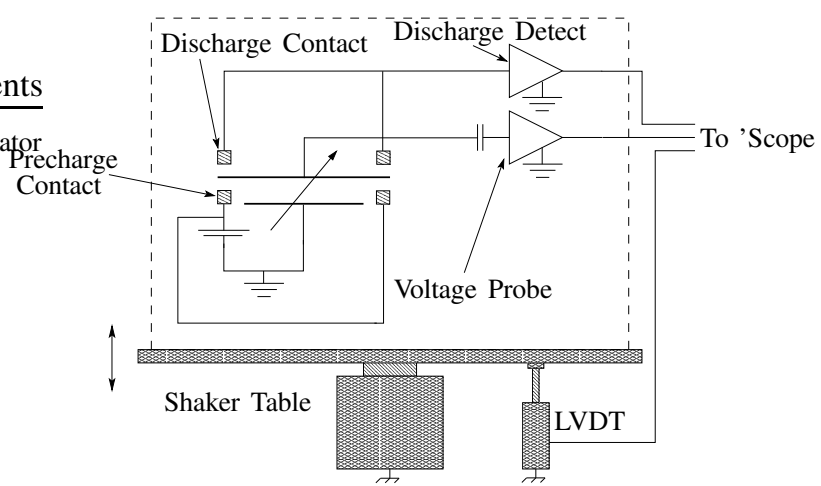

Fig. 6. Experimental setup. Contents of dashed box are mounted on the shaker table.

Measurement of the voltage on the moving plate is non-trivial because of the small capacitance of the generator and the relatively low operating frequency. If a DC-coupled amplifier is used, then the input resistance must be sufficiently high to ensure negligible loss of charge from the generator capacitor during the generation cycle. The required input impedance can be estimated by imposing the condition that the time constant of the generator capacitor and the input resistance be at least 100 times geater than the generator period. For a generator with a minimum capacitance of $4 \mathrm{pF}$ and a period of $50 \mathrm{~ms}$, this leads to an input resistance of at least $10^{12} \Omega$. Operational amplifiers with differential input impedances of $10^{12} \Omega$ are available commercially (9), and these allow very large value resistor networks to be used to set the closed loop gain. For example, a simple inverting amplifer arrangement with a $10^{12} \Omega$ input resistor and a $10^{10} \Omega$ feedback resistor satisfy the biasing requirements and provide a sensible closed loop gain of $\frac{1}{100}$. However, the frequency response of such an amplifier is likely to be dominated by parasitic capacitances associated 
with the large ohmic value resistors. A better approach is to couple the generator voltage into the probe via a capacitor, since it is straightforward to realise a capacitor with a small value compared to the minimum generator capacitance, thereby minimising charge sharing between the generator and the probe, whilst also achieving a leakage resistance $\gg 10^{12} \Omega$.

If the first stage of the capacitively coupled probe is a virtual earth amplifer, then the output of this stage will be proportional to the time derivative of the moving plate voltage. A second stage consisting of an integrator can then be used to yield an output proportional to the voltage on the moving plate. A circuit of this type was constructed with an AD549 electrometer amplifer (9) as a first stage. The performance of this circuit is shown in figure 7, showing the excellent tracking properties of the probe for rates of change of voltage of the order of $50 \mathrm{~V} / \mathrm{ms}$, similar to the rates found in the device. The integrator on the probe is reset on negative going inputs to minimise drift.

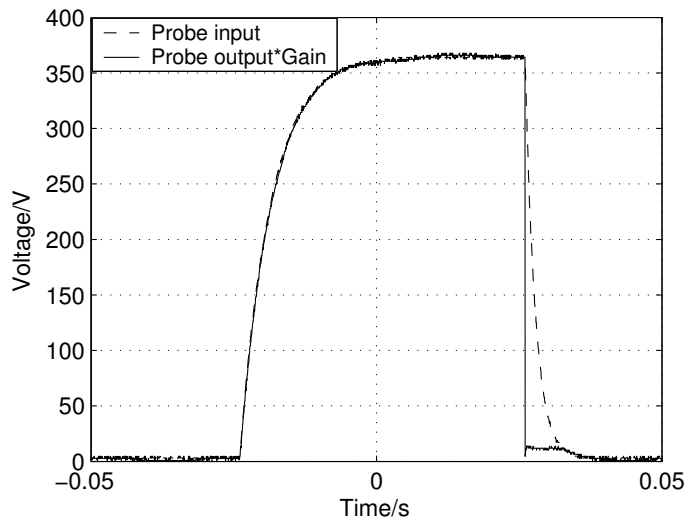

Fig. 7. Transient-tracking test of high-impedance voltage probe.

The discharge circuit was a simple inverting amplifier with an input resistance of $50 \mathrm{M} \Omega$ and a gain of $-\frac{1}{1000}$. This allowed an independent measure of the final voltage on the moving plate at the point of maximum travel. The input resistance was chosen to give a discharge transient of around a millisecond that could easily be observed on an oscilloscope alongside the moving plate voltage.

\section{Prototype Device Fabrication}

A hybrid prototype generator has been fabricated and tested, which has allowed the results of the SPICE simulations to be verified. Figure 8 shows a crosssection of the device, which comprises a silicon proof mass of $0.5 \mathrm{~g}$, supported by flexible polymer membranes, and suspended between a quartz baseplate and a mechanical stop. Deposited gold films on the baseplate and the mass form the fixed and moving plates of the capacitor.

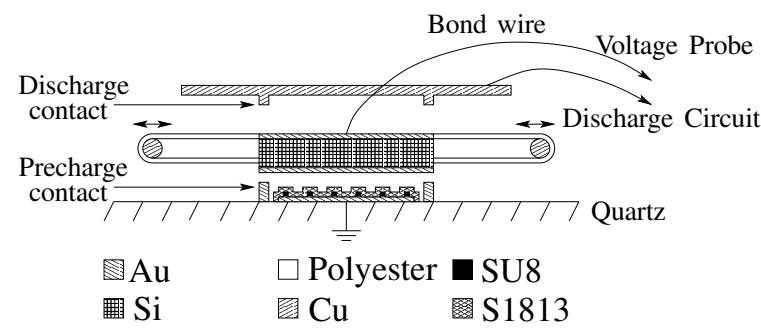

Fig. 8. Cross-section of prototype CFPG.

The quartz baseplate was produced by standard MEMS fabrication techniques. First a quartz wafer was sputter coated with chromium and gold layers which were patterned to form the bottom electrode (fixed plate) which had an active capacitor plate area of $200 \mathrm{~mm}^{2}$ (allowing for the finite area of the charging contacts). An SU8 photoresist layer was then spun and patterned to give an array of $1.5 \mu \mathrm{m}$-high spacer strips covering the electrode area. These were included to limit the possible contact area between the mass and base-plate, and hence reduce the risk of stiction between these two parts in the event that surface curvature brought them into contact. A $1.5 \mu \mathrm{m}$-thick layer of S1813 photoresist was deposited to form a conformal insulating layer over the electrode and spacer strips. Finally, $6 \mu \mathrm{m}$-high electroplated studs were formed on each side of the electrode. These define the minimum capacitor gap, and provide electrical contacts to the moving plate for pre-charging the device at the position of the maximum capacitance.

The proof mass was formed by stacking together three silicon plates, each $10 \mathrm{~mm} \times 11 \mathrm{~mm} \times 400 \mu \mathrm{m}$ in size, with sputtered gold layers on their upper and lower surfaces. The sides of the stack were also met- 

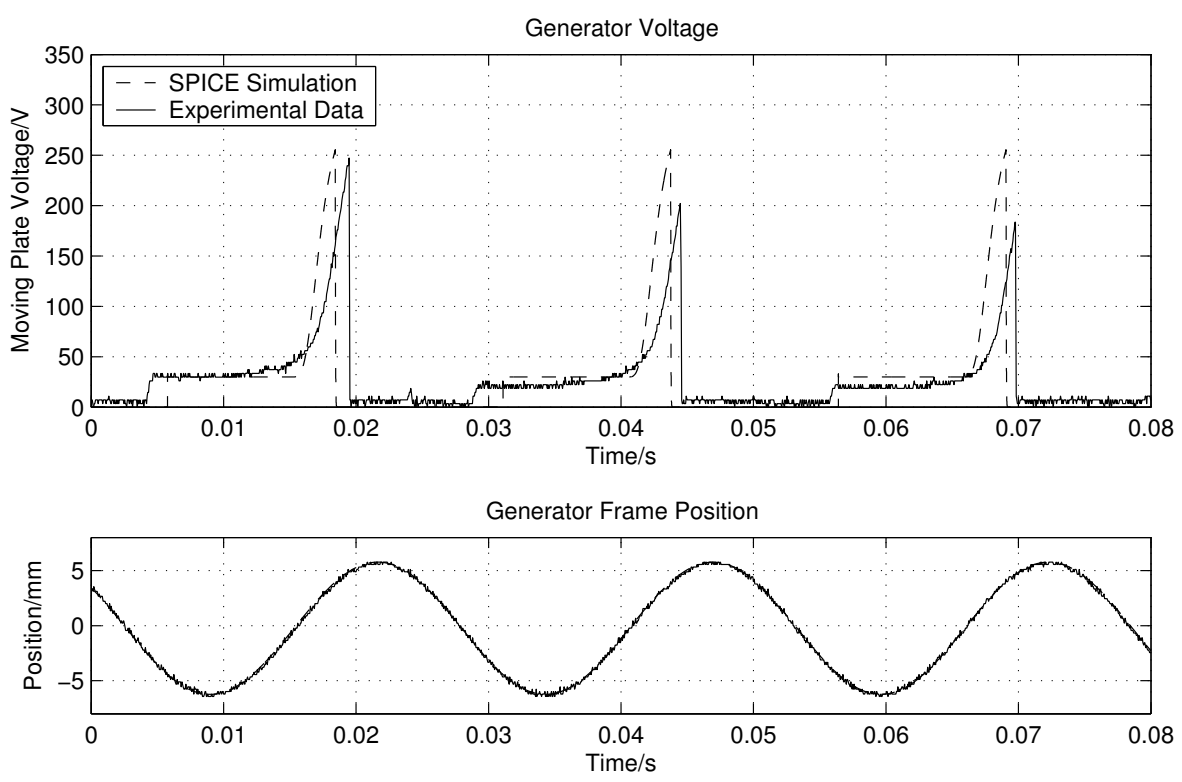

Fig. 9. Simulated results and experimental data.

alised to ensure electrical contact between top and bottom. The suspension consisted of two strips of polyester film, formed into loops. These were bonded on the top of the mass, and also sandwiched between the two lower plates, to form a double flexure suspension at each corner of the mass. The suspension loops were anchored to the baseplate by an adjustable mechanism, allowing a variable amount of pre-loading to be applied. The moving plate was connected to the voltage probe via a thin gold wire, which was bonded to the top surface of the proof mass. A copper mechanical stop was used to limit the travel range of the mass to $\sim 450 \mu \mathrm{m}$ and to provide an additional contact through which the capacitor was discharged at the position of minimum capacitance.

\section{Results}

When the device was mounted on the test rig, the minimum and maximum capacitances of the generator were measured as $15 \mathrm{pF}$ and $127 \mathrm{pF}$. The maximum gap was measured as $450 \mu \mathrm{m}$ which would give a minimum capacitance of $4 \mathrm{pF}$. This suggests that there is an $11 \mathrm{pF}$ parasitic capacitance in parallel with the moving plate, arising from wiring and instrumentation.
The graph of figure 9 shows results from a SPICE simulation of the CFPG compared with the measured data from the device. The data of the generator frame position for the simulation and the experimental data have been aligned. For the ideal behaviour of our prototype CFPG, from a $30 \mathrm{~V}$ precharge, a discharge transient of $900 \mathrm{~V}$ would be observed. When the $11 \mathrm{pF}$ parasitic was added to the SPICE simulation, to yield the results shown in figure 9, the final discharge voltage was found to be $257 \mathrm{~V}$ which is in good agreement with the measurements.

The main difference between the simulation and experimental results is the longer transit time of the moving plate seen in the measured data. This may be due to air damping and squeeze film effects between the plates. The slower than expected initial increase in voltage observed also fits this hypothesis. Another important factor is the orientation of the moving plate during transit. The need for a very low stiffness of the suspension in the direction of travel means that high stiffness for other axes is hard to achieve. The most significant unwanted motion appears to be rotation of the moving plate about the in-plane axes, such that it does not remain parallel to the fixed plate during transit. This alters the holding force and consequently the transit dynamics. 
The simulation results of figure 10 show details of the plate voltage and motion of the mass for parameter values, including parasitics, corresponding to the actual device. Finally, the simulation results in figure 11 show the effect of parasitic capacitance on generator performance, and confirms the importance of keeping the parasitics low.
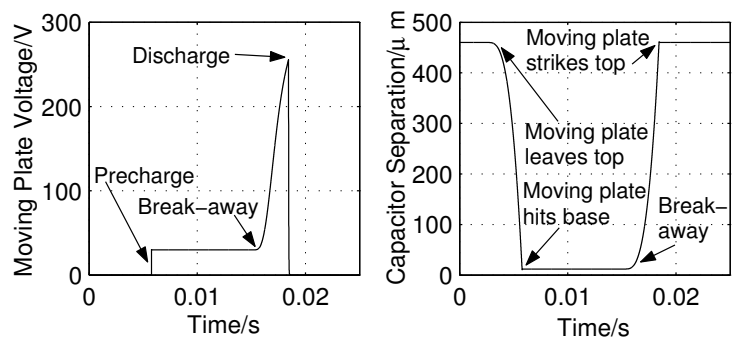

Fig. 10. Detail of plate voltage and plate position.

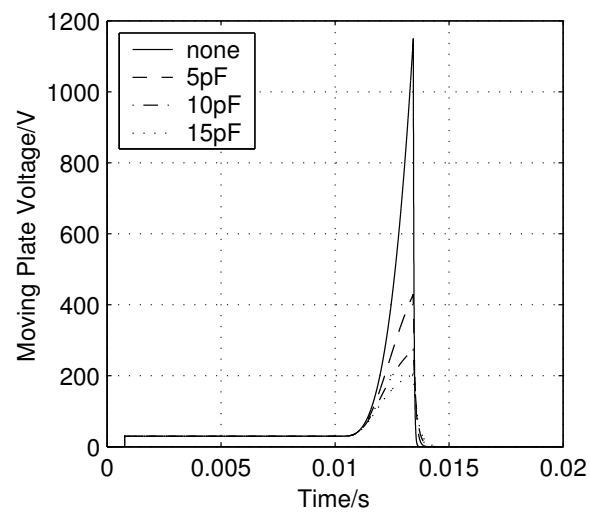

Fig. 11. Effect of parasitic capacitance on the plate voltage.

\section{Conclusions}

An analysis of the dynamics and the maximum energy yield of three architectures of micro-power generator has shown that a non-resonant structure is the best choice for harvesting energy from low frequency, large amplitude movements. An electrostatic generator using an un-sprung proof mass with a non-linear movement has been developed and designated a coulombforce parametric generator. A CFPG prototype has been implemented using a silicon proof mass of $0.5 \mathrm{~g}$ which forms the moving plate of a parallel plate capacitor. The precharging of this capacitor (at minimum separation) sets the frame acceleration at which the mass breaks away and begins to do work. The prototype generates a voltage of $250 \mathrm{~V}$ from a pre-charge of $30 \mathrm{~V}$. Analysis has shown that control of the precharge voltage can be used to optimise the break-away condition so as to maximise the energy harvested. This analysis has been verified against a simulation model and the experimental results. The simulation model includes parasitic components in the system as these can have a large influence on the energy recovered from the very small charges involved.

\section{Acknowledgements}

This project is funded by the EPSRC, and the DC initiative (EU Framework V) under the ORESTEIA project. The authors would also like to thank IMV corporation for loan of equipment.

\section{References}

[1] C. B. Williams, R. B. Yates, Analysis of a Micro-Electric Generator for Microsystems, in: Solid-State Sensors and Actuators, 1995 and Eurosensors IX. Transducers '95. The 8th International Conference on, Vol. 1, 1995, pp. 369-372.

[2] R. Amirtharajah, A. P. Chandrakasan, Self-Powered Signal Processing Using Vibration-Based Power Generation, Solid State Circuits, IEEE Journal of 33 (1998) 687-695.

[3] S. Meninger, J. O. Mur-Miranda, R. Amirtharajah, A. P. Chandrakasan, J. H. Lang, Vibration-to-Electric Energy Conversion, Very Large Scale Integration (VLSI) Systems, IEEE Transactions on 9 (1) (2001) 64-76.

[4] P. Glynne-Jones, S. P. Beeby, E. P. James, N. M. White, The Modelling of a Piezoelectric Vibration Powered Generator for Microsystems, in: Solid-State Sensors and Actuators, the 11th International Conference on, Transducers '01 and Eurosensors XV, Munich, Germany, 2001, pp. 46-49.

[5] W. J. Li, T. C. H. Ho, G. M. H. Chan, P. H. W. Leong, H. Y. Wong, Infrared Signal Transmission by a LaserMicromachined Vibration-Induced Power Generator, in: Circuits and Systems, 2000. Proceedings of the 43rd IEEE Midwest Symposium on, Vol. 1, 2000, pp. 236-239.

[6] R. Tashiro, N. Kabei, K. Katayama, Y. Ishizuka, F. Tsuboi, K. Tsuchiya, Development of an Electrostatic Generator that Harnasses the Motion of a Living Body, JSME International Journal 43 (4) (2000) 916-922, series C.

[7] S. Roundy, P. K. Wright, K. S. Pister, Micro-electrostatic vibration-to-electricity converters, in: 2002 ASME Interna- 
tional Mechanical Engineering Congress and Exposition, New Orleans, Louisiana, USA, 2002.

[8] P. D. Mitcheson, T. C. Green, E. M. Yeatman, A. S. Holmes, Architectures for Vibration-Driven Micro-Power Generators, IEEE/ASME Journal of Micro Electromechanical Systems, in press.

[9] Analog Devices, AD549 Datasheet, Rev. C (October 2002).

\section{Biographies}

Paul D. Mitcheson received the M.Eng. degree in Electrical and Electronic Engineering from Imperial College London, U.K., in 2001. He is currently a Research Assistant in the Control and Power Research Group of that department. He is pursuing the Ph.D. degree focussing on micro-power generators and associated power electronics. He sits on the IEE London Younger Members Committee.

Peng Miao received his B.Eng. degree in electron physics from Zhejiang University, China. He received his MSc degree in chemistry in 1993 from University of Kent at Canterbury, UK and received his $\mathrm{PhD}$ in physics in 1998 from University of Birmingham, UK, specializing in nano-machining and characterization of macro-molecular thin films using STM and HREELS. From 1998 to 2001, Dr Miao worked as a research fellow at Brunel University, UK, in the area of preparation of ceramic thin films using electrostatic atomisation. He is now carrying on his research in power MEMS (design and fabrication of micro-sized power generators) at Imperial College London, UK. Dr Miao has generated nearly 30 publications and a UK patent.

Bernard H. Stark holds the degrees of Diploma in Electrical Engineering from the Swiss Federal Institute of Technology, and Ph.D. in Engineering from Cambridge University. He spent time as a Junior Research Fellow at St. Hughs College in Oxford and is currently a member of the Control and Power Group at Imperial College London. His academic interests include the application of power electronics to energy generation.

Eric M. Yeatman obtained his B.Sc from Dalhousie University, Canada, in 1983, and his Ph.D. from Imperial College London in 1989. Since then he has been a member of staff in the college's Electrical and Electronic Engineering Department, Optical and Semiconductor Devices Group, currently as Reader and Deputy Head of Group. His current research includes micromechanical actuators and generators, microstructures for microwave applications, and integrated optical amplifiers.

Andrew S. Holmes received the B.A. degree in natural sciences from Cambridge University, U.K. in 1987, and the Ph.D. degree in electrical engineering from Imperial College London, U.K. in 1992. He is currently a Senior Lecturer in the Optical and Semiconductor Devices Group, Department of Electrical and Electronic Engineering, Imperial College London. His research interests are in the areas of micropower generation and conversion, MEMS devices for microwave applications, and laser processing for MEMS manufacture.

Tim C. Green received a B.Sc. (Eng) from Imperial College London, UK in 1986 and a Ph.D from Heriot-Watt University, Edinburgh, UK in 1990. Both degrees were in electrical engineering. He was a lecturer at Heriot Watt University until 1994 and is now a Reader at Imperial College London and Deputy Head of the Control and Power Research Group. He has research interests in power electronics applied to generation and distribution of energy including issues of renewable and distributed generation, micro-grids, power quality, active power filters and flexible AC transmission systems. 